\title{
Diversity high up: a cloud forest of the Serra da Mantiqueira as a vascular epiphyte hotspot ${ }^{1}$
}

\author{
Samyra Gomes Furtado ${ }^{2,4}$ \& Luiz Menini Neto ${ }^{2,3}$
}

\begin{abstract}
The diversity of montane environments is dictated by a variety of environmental conditions. Parque Estadual do Ibitipoca is located in the Serra da Mantiqueira, between $\sim 1,000-1,800 \mathrm{~m}$, and harbors approximately 300 ha of cloud forests. The composition of vascular epiphytes was determined by analyzing data from expeditions conducted between July 2014 and July 2015, and specimens deposited at herbaria. The 224 species were distributed into 82 genera of which Pleurothallis s.l. was the richest (13 spp.) and 23 families of which Orchidaceae was the richest (87 spp.). This richness corresponds to approximately $9.5 \%$ of the vascular epiphytic flora of the Atlantic Forest concentrated in an area that comprises $0.00085 \%$ of this phytogeographic domain, which represents one of the largest diversities ever sampled in the Brazilian Atlantic Forest. This fact is more relevant given that 13 species are threatened at the country level and 23 at the state level.
\end{abstract}

Key words: Atlantic Forest, Dwarf-forest, epiphytic quotient, epiphytism, Parque Estadual do Ibitipoca.

\section{Resumo}

A diversidade dos ambientes montanos é condicionada por inúmeras variáveis ambientais. O Parque Estadual do Ibitipoca está localizado na Serra da Mantiqueira, entre $\sim 1.000-1.800$ m, e abriga cerca de 300 ha de florestas nebulares. A composição de epífitas vasculares foi determinada pela análise dos dados de expedições, realizadas entre julho de 2014 e julho de 2015, e espécimes depositados em herbários. Foram registradas 224 espécies distribuídas em 82 gêneros dos quais Pleurothallis s.l. foi o mais rico (13 spp.) e 23 famílias das quais Orchidaceae foi a mais rica (87 spp.). Esta riqueza corresponde a aproximadamente $9,5 \%$ da flora de epífitas vasculares da Floresta Atlântica concentrados em uma área que compreende 0,00085\% deste domínio fitogeográfico, representando uma das maiores diversidades já amostradas na Floresta Atlântica brasileira. Este fato é ainda mais relevante tendo em conta que 13 espécies estão ameaçadas a nível nacional e 23 em nível estadual.

Palavras-chave: Floresta Atlântica, nanofloresta, quociente epifítico, epifitismo, Parque Estadual do Ibitipoca.

\section{Introduction}

Mountainous vegetation harbors a huge diversity of plants due to a combination of factors including isolation (the mountains are comparable to islands), climatic changes that occur in little distances due to the elevational shift, and geodiversity resulting from topographically diverse terrain and differences in substrata (Körner 2004). These factors apply to Parque Estadual do Ibitipoca, in the Serra da Mantiqueira in Minas Gerais, which harbors a remarkable plant richness despite comprising only 1,488 ha (Forzza et al. 2013).
Epiphytes are plants that spend part or all of their life using another plant as support and are of high ecological importance because they provide shelter and resources to animals as well as capturing and storing water (Benzing 1990; Zotz 2016). This synusia also contributes to the diversity, representing $9-10 \%$ of the known vascular species (Gentry \& Dodson 1987; Zotz 2013), with a particular diversity in Neotropical rainforests (Gentry and Dodson 1987). The percentage of vascular epiphytes in the Brazilian Atlantic Forest (BAF) reaches $15 \%$ of the whole domain of

\footnotetext{
${ }^{1}$ Part of Master dissertation of the first author.

${ }^{2}$ Universidade Federal de Juiz de Fora, Inst. Ciências Biológicas, Prog. Pós-graduação em Ecologia (PGECOL/UFJF), Campus Universitário, Bairro Martelos, Juiz de Fora, MG, Brazil.

${ }^{3}$ Universidade Federal de Juiz de Fora, Inst. Ciências Biológicas, Depto. Botânica, Campus Universitário, Bairro Martelos, Juiz de Fora, MG, Brazil.

${ }^{4}$ Author for correspondence: furtadosg@gmail.com
} 
vascular flora, of which 78\% are endemic to Brazil and around $11 \%$ are threatened with extinction. These statistics reinforce the need to acquire knowledge of the biodiversity conservation and ecosystem management practices for this epiphytic group (Freitas et al. 2016).

Studies regarding this epiphytic synusia in Minas Gerais have been intensified (Werneck \& Espírito Santo 2002; Alves et al. 2008; Menini Neto et al. 2009a; Alves \& Menini Neto 2014; Barbosa et al. 2015; Furtado \& Menini Neto 2015a, b, 2016), although they can be considered scarce if taking in account the extension of this state.

A previous checklist of epiphytic angiosperms occurring in PEIB was presented by Menini Neto et al. (2009a), thus the present study aimed to complement the knowledge of vascular epiphytes in PEIB (including ferns as well as new records of angiosperms), an important conservation unit of Minas Gerais (Costa et al. 1998; Drummond et al. 2005; Forzza et al. 2013), contributing to the biodiversity knowledge base of the Serra da Mantiqueira and Atlantic domain.

\section{Material and Methods}

Study site

The Parque Estadual da Serra do Ibitipoca (PEIB) covers approximately 1,488 ha in the southeastern region of Minas Gerais, in the municipality of Lima Duarte, district of Conceição de Ibitipoca between coordinates $21^{\circ} 40^{\prime}-21^{\circ} 44^{\prime} \mathrm{S}$ and $43^{\circ} 52^{\prime}-43^{\circ} 55^{\prime} \mathrm{W}$ (Fig. 1). It is part of the Mantiqueira Mountains (Serra da Mantiqueira) and has an elevational range of 1,000 to $1,784 \mathrm{~m}$. The climate is classified as Cwb according to the Köppen system, with dry winters and mild summers. The averages of annual precipitation and temperature are $1,532 \mathrm{~mm}$ and $18.9^{\circ} \mathrm{C}$, respectively (CETEC 1983).

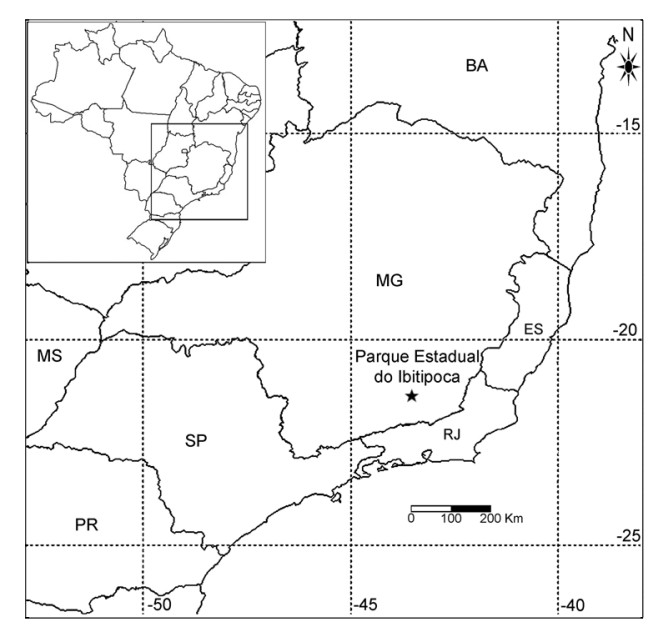

Cloud forest

Cloud Dwarf-forest

Cloud scrub

Cloud parkland savanna and Cloud shrubland savanna (mosaic)

Cloud bushy grassland and cloud grassland (mosaic)

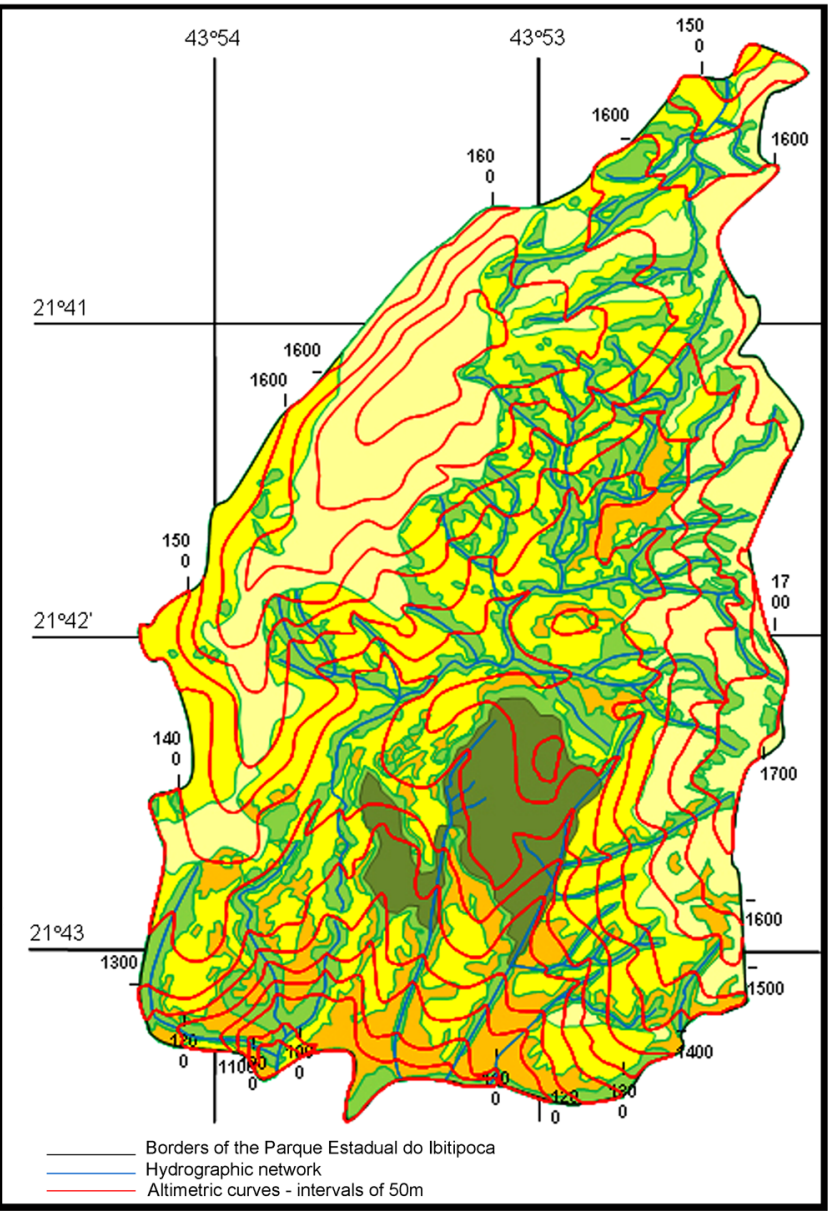

Figure 1 - Location of Parque Estadual do Ibitipoca, Minas Gerais, Brazil. Adapted from Oliveira-Filho et al. (2013). 
The PEIB is situated in the Atlantic domain, and its vegetation harbors a mosaic of field and forest phytophysiognomies, with the predominance of campo rupestre, which has a high floristic richness and several endemic species. Cloud forests are interspersed throughout the field vegetation (covering approximately 300 ha or $20 \%$ of the whole extension of PEIB), especially the so called Mata Grande (with a canopy of $\sim 20 \mathrm{~m}$ ) and the formations of dwarf-forests (with canopy of $\sim 5$ $\mathrm{m})$. In this environment, the richness of vascular epiphytes is remarkable due to the moisture provided by the fog and retained by the trees (Oliveira-Filho et al. 2013) (Fig. 2).

The flora of PEIB is well documented with checklists of bryophytes (hornworts, liverworts, and mosses) (Luizi-Ponzo et al. 2013), ferns and lycophytes (Salino et al. 2013), and spermatophytes (angiosperms and gymnosperms) (Forzza et al. 2013).

\section{Data collection}

We conducted field trips between July 2014 and July 2015 in order to collect fertile specimens (when needed), observe and obtain photographic recordings of epiphytic species. The resulting photographs were published as a rapid color guide of The Field Museum of Chicago ( $<\mathrm{http}$ :// fieldguides.fieldmuseum.org/sites/default/files/ rapid-color-guides-dfs/712_brasil-epifitas_de_ ibitipoca.pdf $>$ ). The collected specimens were deposited at Herbarium CESJ of Universidade Federal de Juiz de Fora. Specimens deposited at the herbaria BHCB, CESJ, HB, R, RB, and VIC (acronyms according to Thiers 2016) from previous collections in the PEIB spanning more than 40 years were also examined.

The evolutionary lineages of vascular plants are according to PPG (2016) for ferns and lycophytes and APG IV (2016) for angiosperms (magnoliids, monocotyledons, and eudicotyledons).

Data about endemism in Brazil were obtained in the database compiled by BFG (2015) (available at <http://dx.doi.org/10.6084/ m9.figshare.1538647>) and Prado et al. (2015). The conservation status of species in Brazil is according to Martinelli \& Moraes (2013), and similar data for Minas Gerais were obtained from Drummond et al. (2008). The distribution of the genera is according to the literature for each group.

Ecological categories are according to Benzing (1990), and we did not differentiate between primary and secondary hemiepiphytes.

\section{Data analysis}

We calculated the epiphytic quotient according to Hosokawa (1950), which represents the percentage of epiphytic plants identified among the vascular species that occur in the cloud forest of PEIB. One calculation set consisted of all the recorded epiphytes, and the other set excluded the accidental ones. This calculation was possible due to the existence of checklists of ferns and lycophytes (Salino et al. 2013) and angiosperms (Forzza et al. 2013 ) in the PEIB. A filtering of species occurring in PEIB cloud forests was conducted, through data obtained in herbarium sheets, literature [especially the published floras for the Park, cited by Forzza et al. (2013)] and field observation.

\section{Results}

A total of 224 species of vascular epiphytes were identified (of which 213 were classified at the specific level) that belonged to 82 genera and 23 families (Tab. 1), including 152 angiosperms [10 magnoliids (4.5\%), $122(54.3 \%)$ monocotyledons and 20 (8.5\%) eudicotyledons], 69 (31.4\%) ferns and 3 lycophytes (1.3\%).

Orchidaceae was the richest family with 86 identified species (38.6\% of the total), followed by Polypodiaceae with 27 species (12.1\%), and Bromeliaceae with 25 (11.2\%) (Fig. 3). The main families of vascular epiphytes recorded in the PEIB are compared with those in some Brazilian ombrophilous forests (Tab. 2). Regarding the genera, Pleurothallis R.Br. sensu lato is the richest with 13 species (5.9\%), Asplenium L. with 11 species (5\%), and Peperomia Ruiz \& Pav. and Epidendrum L. with 10 species each.

Among the ecological categories, characteristic holoepiphytes were classified as the most prominent [127 species (57\%)], followed by facultative holoepiphytes [74 (33.2\%)], accidental holoepiphytes [12 (5.4\%)], and hemiepiphytes [11 $(4.5 \%)]$.

The values of epiphytic quotients were $30 \%$ and $28.5 \%$, respectively, for the total and excluded accidental holoepiphytes.

\section{Discussion}

The region encompassing Ibitipoca State Park has been considered of "Extreme Biological Importance" (Drummond et al. 2005), a fact that is corroborated by the richness of vascular epiphytes. This area represents one of the highest richness levels ever recorded in areas of dense 

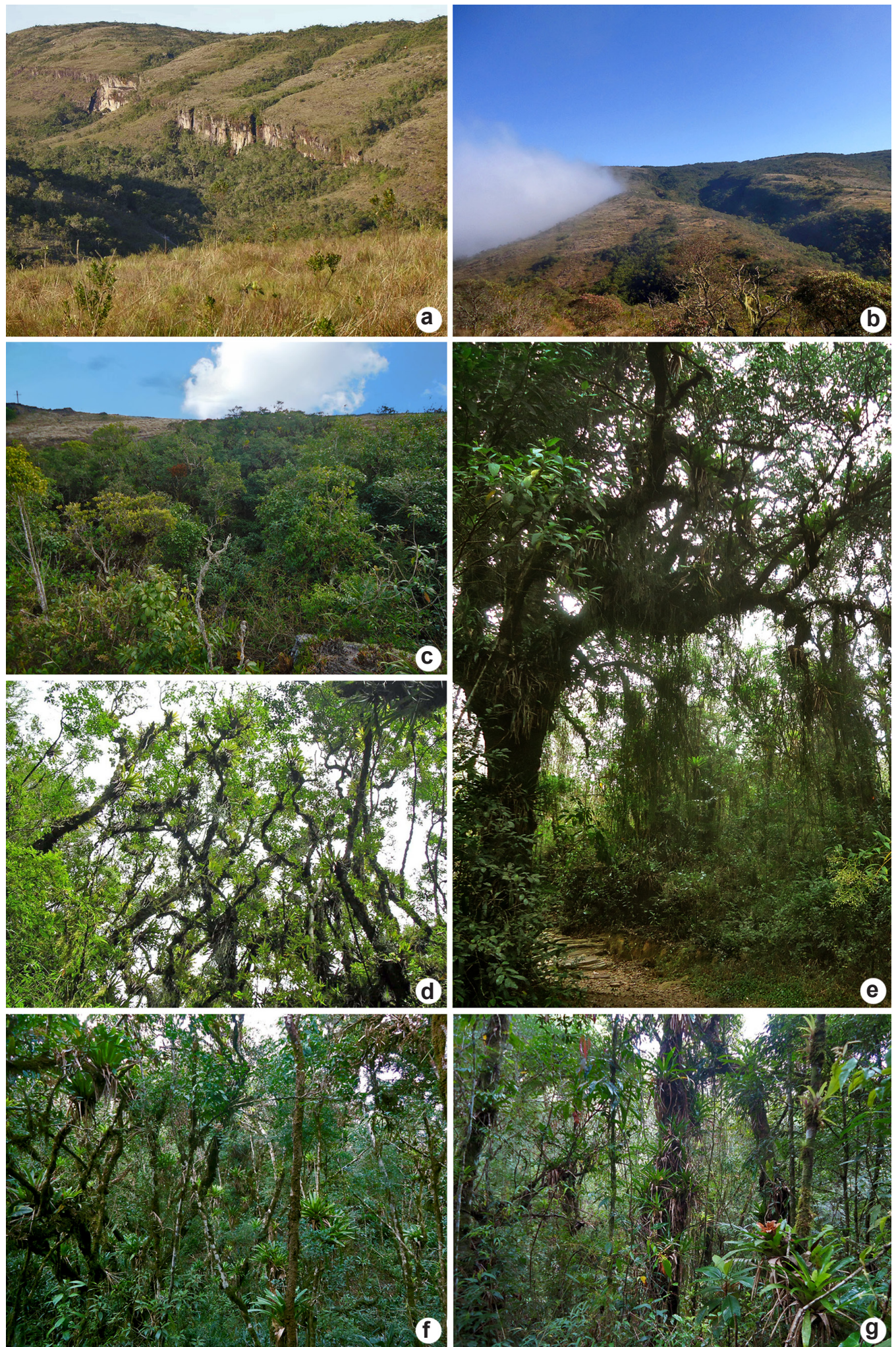

Figure 2 - Vegetation of Parque Estadual do Ibitipoca - a. patches of cloud forest interspersed with campo rupestre; b. detail of fog covering the forests and campo rupestre; c. general view of a cloud forest; d. detail of the canopy of a cloud forest. e-g. interior view of the cloud forests. 
Table 1 - Checklist of vascular epiphyte species recorded in cloud forests of the Parque Estadual do Ibitipoca, Minas Gerais, Brazil.

\begin{tabular}{|c|c|c|c|}
\hline Lineages, families and species & Voucher & CS & EC \\
\hline \multicolumn{4}{|l|}{ Lycophytes (1/3) } \\
\hline \multicolumn{4}{|l|}{ Lycopodiaceae (1/3) } \\
\hline Phlegmariurus biformis (Hook.) B.Øllg. * & L.Krieger (CESJ 2665) & & CHL \\
\hline Phlegmariurus fontinaloides (Spring) B.Øllg. * & L.Krieger (CESJ 9347) & & CHL \\
\hline Phlegmariurus heterocarpon (Fée) B.Øllg. & S.G.Furtado 316 & & CHL \\
\hline \multicolumn{4}{|l|}{ Ferns $(35 / 71)$} \\
\hline \multicolumn{4}{|l|}{ Aspleniaceae $(1 / 10)$} \\
\hline Asplenium auriculatum Sw. & S.G.Furtado 308 & & FHL \\
\hline Asplenium auritum $\mathrm{Sw}$. & R.F.Novelino et al. 842 & & FHL \\
\hline Asplenium claussenii Hieron. & C.M.Mynssen 825 & & FHL \\
\hline Asplenium feei Kunze ex Fee & C.M.Mynssen 824 & & FHL \\
\hline Asplenium geraense (C.Chr.) Sylvestre & S.G.Furtado 309 & & FHL \\
\hline Asplenium harpeodes Kunze & R.F.Novelino et al. 919 & & FHL \\
\hline Asplenium oligophyllum Kaulf. & P.B.Pitta 281 & & FHL \\
\hline Asplenium praemorsum Sw. & S.G.Furtado 319 & & FHL \\
\hline Asplenium pseudonitidum Raddi * & R.C.Forzza 3588 & & FHL \\
\hline Asplenium raddianum Gaudich. & R.C.Forzza 3149 & & FHL \\
\hline Asplenium serra Langsd. \& Fisch. & L.Krieger (CESJ 8383) & & FHL \\
\hline \multicolumn{4}{|l|}{ Blechnaceae $(1 / 1)$} \\
\hline Lomaridium acutum (Desv.) Gasper \& V.A.O. Dittrich & R.F.Novelino 860 & & FHL \\
\hline \multicolumn{4}{|l|}{ Dryopteridaceae (4/11) } \\
\hline Arachniodes denticulata (Sw.) Ching & B.R.Silva 1367 & & AHL \\
\hline Elaphoglossum gayanum (Fée) T.Moore & S.G.Furtado 314 & & FHL \\
\hline Elaphoglossum glabellum J.Sm. & C.M.Mynssen 786 & & FHL \\
\hline Elaphoglossum lingua (C.Presl) Brack. * & R.F.Novelino 1017 & & FHL \\
\hline Elaphoglossum lisboae Rosenst. * & R.F.Novelino 1029 & & $\mathrm{CHL}$ \\
\hline Elaphoglossum luridum (Fee) Christ & R.F.Novelino 1014 & & FHL \\
\hline Elaphoglossum pachydermum (Fée) T.Moore * & J.E.Z.Oliveira 217 & & AHL \\
\hline Elaphoglossum strictum (Raddi) T.Moore & J.E.Z.Oliveira 398 & & CHL \\
\hline Elaphoglossum vagans (Mett.) Hieron. * & R.F.Novelino 1188 & & FHL \\
\hline Polybotrya speciosa Schott * & C.M.Mynssen 828 & & Hem \\
\hline Rumohra adiantiformis (G.Forst.) Ching & L.Menini Neto 1354 & & FHL \\
\hline \multicolumn{4}{|l|}{ Hymenophylaceae (4/14) } \\
\hline Didymoglossum hymenoides (Hedw.) Desv. & L.Krieger (BHCB 4220) & DD MG & $\mathrm{CHL}$ \\
\hline Didymoglossum krausii (Hook. \& Grev.) C.Presl & L.Krieger (CESJ 18823) & & CHL \\
\hline Hymenophyllum crispum Kunth & T.E.Almeida 1171 & & CHL \\
\hline
\end{tabular}




\begin{tabular}{|c|c|c|c|}
\hline Lineages, families and species & Voucher & CS & $\mathbf{E C}$ \\
\hline Hymenophyllum fragile (Hedw.) C.V.Morton & T.E.Almeida 1186 & & CHL \\
\hline Hymenophyllum fucoides (Sw.) Sw. & T.E.Almeida 1241 & & CHL \\
\hline Hymenophyllum hirsutum (L.) Sw. & L.Krieger (CESJ 8375) & & FHL \\
\hline Hymenophyllum polyanthos (Sw.) Sw. & L.Krieger (CESJ 3549) & & $\mathrm{CHL}$ \\
\hline Hymenophyllum pulchellum Schltdl. \& Cham. & T.E.Almeida 1167 & & CHL \\
\hline Hymenophyllum sp. & S.G.Furtado 289 & & CHL \\
\hline Polyphlebium angustatum (Carmich.) Ebihara \& Dubuisson & C.M.Mynssen 831 & & CHL \\
\hline Polyphlebium diaphanum (Kunth) Ebihara \& Dubuisson * & L.Krieger (CESJ 16218) & & FHL \\
\hline Polyphlebium hymenophylloides (Bosch) Ebihara \& Dubuisson & T.E.Almeida 1181 & & CHL \\
\hline Trichomanes pilosum Raddi & R.F.Novelino 870 & & AHL \\
\hline Trichomanes polypodioides Raddi & C.M.Mynssen 779 & & $\mathrm{CHL}$ \\
\hline \multicolumn{4}{|l|}{ Nephrolepidaceae (1/1) } \\
\hline Nephrolepis sp. & Not collected & & AHL \\
\hline \multicolumn{4}{|l|}{ Polypodiaceae (12/27) } \\
\hline Campyloneurum angustifolium (Sw.) Fee & R.C.Forzza 3152 & & CHL \\
\hline Campyloneurum nitidum (Kaulf.) C.Presl & S.G.Furtado 310 & & FHL \\
\hline Campyloneurum phyllitidis (L.) C.Pres1 & J.E.Z.Oliveira 228 & & $\mathrm{CHL}$ \\
\hline Cochlidium punctatum (Raddi) L.E.Bishop * & L.Krieger (CESJ 11674) & & CHL \\
\hline Cochlidium serrulatum (Sw.) L.E.Bishop & S.G.Furtado 294 & & FHL \\
\hline Lellingeria apiculata (Kunze ex Klotzsch) A.R.Sm. \& R.C.Moran & L.Krieger (CESJ 64862) & & $\mathrm{CHL}$ \\
\hline Leucotrichum sp. & Not collected & & $\mathrm{CHL}$ \\
\hline Melpomene flabelliformis (Poir.) A.R.Sm. \& R.C.Moran * & L.Krieger (CESJ 15133) & & CHL \\
\hline Melpomene peruviana (Desv.) A.R.Sm. \& R.C.Moran & L.Krieger (CESJ 8380) & CR MG & FHL \\
\hline Melpomene pilosissima (M.Martens \& Galeotti) A.R.Sm. \& R.C.Moran & S.G.Furtado 288 & & $\mathrm{CHL}$ \\
\hline Microgramma percussa (Cav.) de la Sota & L.Krieger (CESJ 21276) & & FHL \\
\hline Microgramma squamulosa (Kaulf.) de la Sota & R.F.Novelino 933 & & FHL \\
\hline Moranopteris achilleifolia (Kaulf.) R.Y. Hirai \& J. Prado * & S.G.Furtado 287 & & $\mathrm{CHL}$ \\
\hline Moranopteris gradata (Baker) R.Y. Hirai \& J. Prado * & R.C.Forzza 4436 & & FHL \\
\hline Niphidium crassifolium (L.) Lellinger & S.G.Furtado 323 & & FHL \\
\hline Pecluma pectinatiformis (Lindm.) M.G.Price & C.M.Mynssen 788 & & $\mathrm{CHL}$ \\
\hline Pecluma recurvata (Kaulf.) M.G.Price * & R.C.Forzza 3158 & & CHL \\
\hline Pecluma truncorum (Lindm.) M.G.Price * & C.M.Mynssen 833 & & CHL \\
\hline Phlebodium pseudoaureum (Cav.) Lellinger & R.C.Forzza 3587 & & FHL \\
\hline Pleopeltis astrolepis (Liebm.) E.Fourn. & S.G.Furtado 285 & & CHL \\
\hline Pleopeltis hirsutissima (Raddi) de la Sota & L.Krieger (CESJ 27419) & & CHL \\
\hline Pleopeltis macrocarpa (Bory ex Willd.) Kaulf. & R.F.Novelino 1263 & & CHL \\
\hline Pleopeltis minarum (Weath.) Salino * & R.C.Forzza 3715 & & CHL \\
\hline Serpocaulon catharinae (Langsd. \& Fisch.) A.R.Sm. * & D.Sucre 6737 & & FHL \\
\hline
\end{tabular}




\begin{tabular}{|c|c|c|c|}
\hline Lineages, families and species & Voucher & CS & $\mathbf{E C}$ \\
\hline Serpocaulon fraxinifolium (Jacq.) A.R.Sm. & J.E.Z.Oliveira 375 & & Hem \\
\hline Serpocaulon latipes (Langsd. \& Fisch.) A.R.Sm. * & L.Krieger (CESJ 8378) & & AHL \\
\hline Polypodiaceae indet. & S.G.Furtado 292 & & CHL \\
\hline \multicolumn{4}{|l|}{ Pteridaceae $(2 / 4)$} \\
\hline Radiovittaria gardneriana (Fée) E.H.Crane & C.M.Mynssen 813 & & CHL \\
\hline Radiovittaria stipitata (Kunze) E.H.Crane & J.E.Z.Oliveira 397 & & FHL \\
\hline Vittaria graminifolia Kaulf. & S.G.Furtado 315 & & FHL \\
\hline Vittaria lineata (L.) Sm. & R.F.Novelino 59 & & FHL \\
\hline \multicolumn{4}{|l|}{ Angiosperms } \\
\hline \multicolumn{4}{|l|}{ Magnoliids (1/10) } \\
\hline \multicolumn{4}{|l|}{ Piperaceae $(1 / 10)$} \\
\hline Peperomia augescens Miq. \#* & F.Salimena (CESJ 27411) & & AHL \\
\hline Peperomia corcovadensis Gardn. \# & D.Monteiro 625 & & CHL \\
\hline Peperomia crinicaulis C.DC.\#* & S.G.Furtado 291 & & CHL \\
\hline Peperomia decora Dahlst. * (MG) & L.Krieger (CESJ 8541) & & FHL \\
\hline Peperomia diaphanoides Dahlst. \# & S.G.Furtado 306 & & FHL \\
\hline Peperomia galioides Kunth \# & S.G.Furtado 303 & & FHL \\
\hline Peperomia loxensis Kunth \# & D.Sucre 7246 & & CHL \\
\hline Peperomia mandioccana Miq. * & S.G.Furtado 302 & & FHL \\
\hline Peperomia rotundifolia (L.) Kunth \# & H.Magalhães (R 86560) & & CHL \\
\hline Peperomia tetraphylla (G.Forst.) Hook. \& Arn. & L.Krieger (CESJ 16238) & & CHL \\
\hline \multicolumn{4}{|l|}{ Monocotyledons (42/120) } \\
\hline \multicolumn{4}{|l|}{ Amaryllidaceae (1/1) } \\
\hline Hippeastrum aulicum (Ker Gawl.) Herb. \#* & P.M.Andrade (BHCB 92378) & & FHL \\
\hline \multicolumn{4}{|l|}{ Araceae (2/9) } \\
\hline Anthurium boudetii Nadruz * & L.Temponi 397 & & FHL \\
\hline Anthurium comtum Schott * & L.Temponi 400 & & FHL \\
\hline Anthurium leonii E.G.Gonç. * (MG) & R.C.Forzza 4270 & CR MG & FHL \\
\hline Anthurium minarum Sakur. \& Mayo * & L.Temponi 390 & & FHL \\
\hline Anthurium scandens (Aubl.) Engl. & R.C.Forzza 2663 & & CHL \\
\hline Philodendron appendiculatum Nadruz \& Mayo * & R.C.Forzza 3638 & & Hem \\
\hline Philodendron bipinnatifidum Schott & L.Temponi 410 & & Hem \\
\hline Philodendron minarum Engl. * & R.C.Forzza 2653 & & Hem \\
\hline Philodendron propinquum Schott * & L.Temponi 398 & & Hem \\
\hline \multicolumn{4}{|l|}{ Bromeliaceae $(8 / 25)$} \\
\hline Aechmea bromeliifolia (Rudge) Baker \# & E.M.C.Leme 1474 & & $\mathrm{CHL}$ \\
\hline Aechmea nudicaulis (L.) Griseb. * & R.Monteiro 13 & & CHL \\
\hline Billbergia alfonsijoannis Reitz * & E.M.C.Leme 1475 & & CHL \\
\hline
\end{tabular}




\begin{tabular}{|c|c|c|c|}
\hline Lineages, families and species & Voucher & CS & EC \\
\hline Billbergia distachia (Vell.) Mez\#* & S.G.Furtado 325 & & FHL \\
\hline Neoregelia ibitipocensis (Leme) Leme * & R.C.Forzza 3338 & CR MG & FHL \\
\hline Neoregelia lymaniana R.Braga \& Sucre * & E.M.C.Leme 1478 & EN MG & FHL \\
\hline Neoregelia oligantha L.B.Sm. * (MG) & R.F.Monteiro 38 & $\begin{array}{l}\text { VU BR } \\
\text { EN MG }\end{array}$ & FHL \\
\hline Nidularium ferdinandocoburgii Wawra * & R.C.Forzza 3182 & & FHL \\
\hline Nidularium marigoi Leme * & R.C.Forzza 3232 & $\begin{array}{l}\text { NT BR } \\
\text { VU MG }\end{array}$ & FHL \\
\hline Racinaea aerisincola $(\mathrm{Mez})$ M.A.Spencer \& L.B.Sm. * & D.Sucre 7147 & & CHL \\
\hline Tillandsia gardneri Lindl. & R.C.Forzza 3094 & & $\mathrm{CHL}$ \\
\hline Tillandsia geminiflora Brongn. & L.Krieger (CESJ 8595) & & CHL \\
\hline Tillandsia recurvata (L.) L. & D.Sucre 7234 & & $\mathrm{CHL}$ \\
\hline Tillandsia streptocarpa Baker \# & L.Menini Neto 1349 & & AHL \\
\hline Tillandsia stricta Sol. & S.G.Furtado 318 & & $\mathrm{CHL}$ \\
\hline Tillandsia tenuifolia $\mathrm{L}$. & R.C.Forzza 3137 & & $\mathrm{CHL}$ \\
\hline Tillandsia usneoides (L.) L. & S.G.Furtado 317 & & $\mathrm{CHL}$ \\
\hline Vriesea bituminosa Wawra * & R.F.Monteiro 28 & & FHL \\
\hline Vriesea carinata Wawra * & C.C.Paula (VIC 26470) & & $\mathrm{CHL}$ \\
\hline Vriesea friburgensis $\mathrm{Mez} *$ & E.M.C.Leme 1473 & & FHL \\
\hline Vriesea guttata Linden \& André * & R.F.Monteiro 25 & & CHL \\
\hline Vriesea heterostachys (Baker) L.B.Sm. * & G.Martinelli 15301 & & $\mathrm{CHL}$ \\
\hline Vriesea longicaulis (Baker) Mez * & G.Martinelli 15314 & & FHL \\
\hline Vriesea penduliflora L.B.Sm. * & E.M.C.Leme 1476 & $\begin{array}{l}\text { EN BR } \\
\text { VU MG }\end{array}$ & FHL \\
\hline Wittrockia gigantea (Baker) Leme * & G.Martinelli 15313 & & FHL \\
\hline \multicolumn{4}{|l|}{ Commelinaceae (1/1) } \\
\hline Commelina obliqua Vahl \# & L.Menini Neto 1355 & & AHL \\
\hline \multicolumn{4}{|l|}{ Orchidaceae $(30 / 86)$} \\
\hline Bifrenaria aureofulva Lindl. \#* & R.C.Forzza 88 & & FHL \\
\hline Bifrenaria harrisoniae (Hook.) Rchb.f. \#* & P.I.S.Braga 1938 & & FHL \\
\hline Bifrenaria stefanae V.P.Castro \#* & H.C.Souza (BHCB 9082) & & $\mathrm{CHL}$ \\
\hline Bifrenaria vitellina (Lindl.) Lindl. * & L.Menini Neto 35 & EN MG & $\mathrm{CHL}$ \\
\hline Bulbophyllum micropetaliforme J.E.Leite * & L.Menini Neto 152 & & CHL \\
\hline Bulbophyllum exaltatum Lindl. \# & D.Sucre 6839 & & FHL \\
\hline Bulbophyllum glutinosum (Barb.Rodr.) Cogn. * & L.Menini Neto 125 & & $\mathrm{CHL}$ \\
\hline Bulbophyllum granulosum Barb.Rodr. * & L.Menini Neto 107 & & CHL \\
\hline Bulbophyllum regnellii Rchb.f. & L.Menini Neto 124 & & CHL \\
\hline Campylocentrum cf. neglectum (Rchb.f. \& Warm.) Cogn. & L.Menini Neto 28 & & $\mathrm{CHL}$ \\
\hline Campylocentrum cf. robustum Cogn. $*$ & L.Menini Neto 193 & & CHL \\
\hline Cattleya bicolor Lindl. * & L.Menini Neto 178 & $\begin{array}{l}\text { NT BR } \\
\text { VU MG }\end{array}$ & $\mathrm{CHL}$ \\
\hline
\end{tabular}




\begin{tabular}{|c|c|c|c|}
\hline Lineages, families and species & Voucher & CS & EC \\
\hline Cattleya loddigesii Lindl. * & $\begin{array}{l}\text { Without collector (CESJ } \\
27534)\end{array}$ & EN MG & $\mathrm{CHL}$ \\
\hline Centroglossa macroceras Rchb.f. * & R.C.Forzza 54 & & CHL \\
\hline Dichaea cogniauxiana Schltr. * & L.Menini Neto 142 & & $\mathrm{CHL}$ \\
\hline Elleanthus brasiliensis (Lindl.) Rchb.f.\# & L.Menini Neto 126 & & FHL \\
\hline Encyclia patens Hook. * & S.G.Furtado 299 & & CHL \\
\hline Epidendrum armeniacum Lindl. & L.Menini Neto 175 & & $\mathrm{CHL}$ \\
\hline Epidendrum avicula Lindl. \# & M.C.Brügger (CESJ 24693) & & CHL \\
\hline Epidendrum chlorinum Barb.Rodr. * & L.Menini Neto 171 & & CHL \\
\hline Epidendrum cf. filicaule Lindl. \# & L.Menini Neto 1350 & & CHL \\
\hline Epidendrum pseudodifforme Hoehne \& Schltr. * & L.Menini Neto 97 & & CHL \\
\hline Epidendrum ochrochlorum Barb.Rodr. * & S.G.Furtado 297 & EN MG & $\mathrm{CHL}$ \\
\hline Epidendrum paranaense Barb.Rodr. * & L.Menini Neto 131 & & FHL \\
\hline Epidendrum ramosum Jacq. \# & R.C.Forzza 16 & & FHL \\
\hline Epidendrum rigidum Jacq. & L.Menini Neto 71 & & $\mathrm{CHL}$ \\
\hline Epidendrum secundum Jacq. \# & L. Menini Neto 46 & & FHL \\
\hline Eurystyles actinosophila (Barb.Rodr.) Schltr. \# & S.G. Furtado 326 & & CHL \\
\hline Eurystyles cogniauxii (Kraenzl.) Pabst* & L.Menini Neto 77 & & $\mathrm{CHL}$ \\
\hline Gomesa glaziovii Cogn. * & L.Menini Neto 76 & & FHL \\
\hline Gomesa gomezoides (Barb.Rodr.) Pabst* & L.Menini Neto 30 & & $\mathrm{CHL}$ \\
\hline Gomesa recurva R.Br. & L.Menini Neto 154 & & CHL \\
\hline Grobya amherstiae Lindl. * & R.C.Forzza 26 & & $\mathrm{CHL}$ \\
\hline Hadrolaelia coccinea (Lindl.) Chiron \& V.P.Castro & L.Menini Neto 161 & EN MG & $\mathrm{CHL}$ \\
\hline Hoffmannseggella crispata (Thunb.) H.G.Jones \#* (MG) & Not collected & $\begin{array}{l}\text { NT BR } \\
\text { EN MG }\end{array}$ & AHL \\
\hline Isabelia violacea (Lindl.) van den Berg \& M.W.Chase * & S.G.Furtado 282 & & $\mathrm{CHL}$ \\
\hline Isabelia virginalis Barb.Rodr. & L.Menini Neto 47 & VU BR & $\mathrm{CHL}$ \\
\hline Isochilus linearis (Jacq.) R.Br & L.Menini Neto 44 & & $\mathrm{CHL}$ \\
\hline Lankesterella gnoma (Kraenzl.) Hoehne * & L.Menini Neto 139 & & $\mathrm{CHL}$ \\
\hline Masdevallia infracta Lindl. & L.Menini Neto 173 & & $\mathrm{CHL}$ \\
\hline Maxillaria brasiliensis Brieger \& Illg \#* & L.Menini Neto 88 & & FHL \\
\hline Maxillaria gracilis Lodd. \#* & L.C.S.Assis 1080 & & FHL \\
\hline Maxillaria notylioglossa Rchb.f. & L.Menini Neto 119 & & $\mathrm{CHL}$ \\
\hline Maxillaria ochroleuca Lodd. ex Lindl. & L.Menini Neto 87 & & $\mathrm{CHL}$ \\
\hline Maxillaria picta Hook. \# & R.C.Forzza 92 & & FHL \\
\hline Maxillaria subulata Lindl. * & L.Menini Neto 48 & & FHL \\
\hline Octomeria crassifolia Lindl. & L.Menini Neto 138 & & CHL \\
\hline Octomeria diaphana Lindl. * & L.Menini Neto 111 & & $\mathrm{CHL}$ \\
\hline Octomeria grandiflora Lindl. & S.G.Furtado 300 & & CHL \\
\hline
\end{tabular}




\begin{tabular}{|c|c|c|c|}
\hline Lineages, families and species & Voucher & CS & EC \\
\hline Octomeria rubrifolia Barb.Rodr. * & L.Menini Neto 40 & & $\mathrm{CHL}$ \\
\hline Octomeria wawrae Rchb.f. * & L.Menini Neto 168 & EN BR & $\mathrm{CHL}$ \\
\hline Oncidium divaricatum Lindl. * & R.C.Forzza 2190 & VU BR & $\mathrm{CHL}$ \\
\hline Oncidium gravesianum Rolfe * & L.Menini Neto 112 & & $\mathrm{CHL}$ \\
\hline Oncidium hookeri Rolfe * & L.Menini Neto 96 & & $\mathrm{CHL}$ \\
\hline Oncidium longipes Lindl. & L.Menini Neto 163 & & $\mathrm{CHL}$ \\
\hline Oncidium truncatum Pabst* & L.Menini Neto 95 & CR BR & $\mathrm{CHL}$ \\
\hline Oncidium warmingii Rchb.f.\# & G.Martinelli 15300 & VU MG & AHL \\
\hline Pleurothallis cryptophoranthoides Loefgr. * & L.Menini Neto 176 & EN MG & $\mathrm{CHL}$ \\
\hline Pleurothallis heliconiscapa Hoehne \#* & H.C.Sousa (BHCB 9833) & & $\mathrm{CHL}$ \\
\hline Pleurothallis hypnicola Lindl. & L.Menini Neto 134 & & $\mathrm{CHL}$ \\
\hline Pleurothallis liparanges Rchb.f.* & L.Menini Neto 177 & CR MG & $\mathrm{CHL}$ \\
\hline Pleurothallis luteola Lindl. & L.Menini Neto 158 & & $\mathrm{CHL}$ \\
\hline Pleurothallis malachantha Rchb.f. * & L.Menini Neto 90 & VU MG & $\mathrm{CHL}$ \\
\hline Pleurothallis marginalis Rchb.f. & L.Menini Neto 162 & & $\mathrm{CHL}$ \\
\hline Pleurothallis quartzicola (Barb.Rodr.) Cogn. * & S.G.Furtado 283 & & $\mathrm{CHL}$ \\
\hline Pleurothallis recurva Lindl. & L.Menini Neto 236 & & $\mathrm{CHL}$ \\
\hline Pleurothallis rubens Lindl. & L.Menini Neto 31 & & $\mathrm{CHL}$ \\
\hline Pleurothallis saundersiana Rchb.f. * & L.Menini Neto 37 & & $\mathrm{CHL}$ \\
\hline Pleurothallis cf. saurocephala Lodd. \#* & Not collected & & $\mathrm{CHL}$ \\
\hline Pleurothallis tricarinata Poepp. \& Endl. & L.Menini Neto 118 & & $\mathrm{CHL}$ \\
\hline Polystachya hoehneana Kraenzl. * & L.Menini Neto 91 & VU MG & $\mathrm{CHL}$ \\
\hline Polystachya estrellensis Rchb.f. \#* & L.Menini Neto 1348 & & $\mathrm{CHL}$ \\
\hline Prescottia stachyodes (Sw.) Lindl. \# & D.R.Gonzaga 43 & & AHL \\
\hline Promenaea xanthina (Lindl.) Lindl. * & L.Menini Neto 130 & & $\mathrm{CHL}$ \\
\hline Prosthechea allemanoides (Hoehne) W.E.Higgins * & L.Menini Neto 26 & & FHL \\
\hline Prosthechea calamaria (Lindl.) W.E.Higgins * & L.Menini Neto 180 & & $\mathrm{CHL}$ \\
\hline Prosthechea pachysepala (Klotzsch) Chiron \& V.P.Castro * & L.Menini Neto 36 & & FHL \\
\hline Prosthechea aff. pachysepala (Klotzsch) Chiron \& V.P.Castro \# & D.R.Gonzaga 44 & & $\mathrm{CHL}$ \\
\hline Scaphyglottis modesta (Rchb.f.) Schltr. & L.Menini Neto 52 & & FHL \\
\hline Scuticaria hadwenii (Lindl.) Planch. * & R.C.Forzza 15 & EN MG & $\mathrm{CHL}$ \\
\hline Stelis aprica Lindl. & L.Menini Neto 127 & & $\mathrm{CHL}$ \\
\hline Stelis intermedia Poepp. \& Endl. & L.Menini Neto 159 & & $\mathrm{CHL}$ \\
\hline Stelis megantha Barb.Rodr. * & L.Menini Neto 148 & & $\mathrm{CHL}$ \\
\hline Stelis papaquerensis Rchb.f. & L.Menini Neto 157 & & $\mathrm{CHL}$ \\
\hline Stelis aff. caespitosa Lindl. & L.Menini Neto 25 & & $\mathrm{CHL}$ \\
\hline Thysanoglossa organensis Brade * & L.Menini Neto 89 & & $\mathrm{CHL}$ \\
\hline
\end{tabular}




\begin{tabular}{|c|c|c|c|}
\hline Lineages, families and species & Voucher & CS & $\mathbf{E C}$ \\
\hline \multicolumn{4}{|l|}{ Eudicotiledôneas (13/19) } \\
\hline \multicolumn{4}{|l|}{ Begoniaceae $(1 / 2)$} \\
\hline Begonia angulata Vell. * & S.G.Furtado 321 & & FHL \\
\hline Begonia sp. & R.C.Forzza 4287 & & FHL \\
\hline \multicolumn{4}{|l|}{ Cactaceae $(5 / 9)$} \\
\hline Arthrocereus melanurus (K.Schum.) Diers et al. subsp. magnus N.P.Taylor & Not collected & EN BR & AHL \\
\hline \& Zappi \#* (MG) & & CR MG & \\
\hline Hatiora salicornioides (Haw.) Britton \& Rose * & M.C.Brügger (CESJ 21541) & & FHL \\
\hline Lepismium cruciforme (Vell.) Miq. \# & L.Menini Neto 1351 & & CHL \\
\hline Lepismium houlletianum (Lem.) Barthlott & S.G.Furtado 313 & & CHL \\
\hline Rhipsalis elliptica G.Lindb. ex K.Schum. * & R.C.Forzza 3226 & & $\mathrm{CHL}$ \\
\hline Rhipsalis floccosa Salm-Dyck ex Pfeiff. & L.Krieger (CESJ 8589) & & $\mathrm{CHL}$ \\
\hline Rhipsalis juengeri Barthlott \& N.P.Taylor * & L.Krieger (CESJ 8594) & & $\mathrm{CHL}$ \\
\hline Rhipsalis pulchra Loefgr. * & L.Krieger (CESJ 9296) & & $\mathrm{CHL}$ \\
\hline Schlumbergera opuntioides (Loefgr. \& Dusén) D.R.Hunt * & D.C.Zappi 258 & $\begin{array}{l}\text { VU BR } \\
\text { VU MG }\end{array}$ & FHL \\
\hline \multicolumn{4}{|l|}{ Gesneriaceae $(2 / 3)$} \\
\hline Nematanthus crassifolius (Schott) Wiehler * & R.C.Forzza 4274 & VU MG & $\mathrm{CHL}$ \\
\hline Nematanthus strigillosus (Mart.) H.E.Moore \#* & L.Krieger (CESJ 13168) & NT BR & FHL \\
\hline Sinningia magnifica (Otto \& A.Dietr.) Wiehler * & R.C.Forzza (CESJ 27323) & & FHL \\
\hline \multicolumn{4}{|l|}{ Griseliniaceae (1/1) } \\
\hline Griselinia ruscifolia (Clos) Taub. & S.G.Furtado 322 & NT BR & Hem \\
\hline \multicolumn{4}{|l|}{ Lentibulariaceae $(1 / 1)$} \\
\hline Utricularia reniformis A.St.-Hil. \#* & R.C.Forzza 3095 & & CHL \\
\hline \multicolumn{4}{|l|}{ Moraceae $(1 / 1)$} \\
\hline Ficus cf. mexiae Standl. \#* & Not collected & & Hem \\
\hline \multicolumn{4}{|l|}{ Rubiaceae (1/1) } \\
\hline Hillia parasitica Jacq. & L.Menini Neto 1353 & & FHL \\
\hline \multicolumn{4}{|l|}{ Solanaceae $(1 / 1)$} \\
\hline Dyssochroma viridiflorum (Sims) Miers \#* & S.G.Furtado 301 & & Hem \\
\hline \multicolumn{4}{|l|}{ Urticaceae $(1 / 1)$} \\
\hline Coussapoa microcarpa (Schott) Rizzini \# & not collected & & Hem \\
\hline
\end{tabular}

The number of genera and species recorded is indicated within parentheses after the lineages and families. EC: Ecological category; Hem: Hemiepiphyte; AHL: Accidental holoepiphyte; CHL: Characteristic holoepiphyte; FHL: Facultative holoepiphyte. CS: Conservation status: CR: Critically endangered; DD: Data deficient; EN: Endangered; NT: Near threatened; VU: Vulnerable. MG: Minas Gerais; BR: Brazil. In the checklist of angiosperms, the species not recorded by Menini Neto et al. (2009a) are marked with \#. The species marked with* are endemic to Brazil (BFG 2015; Prado et al. 2015). 


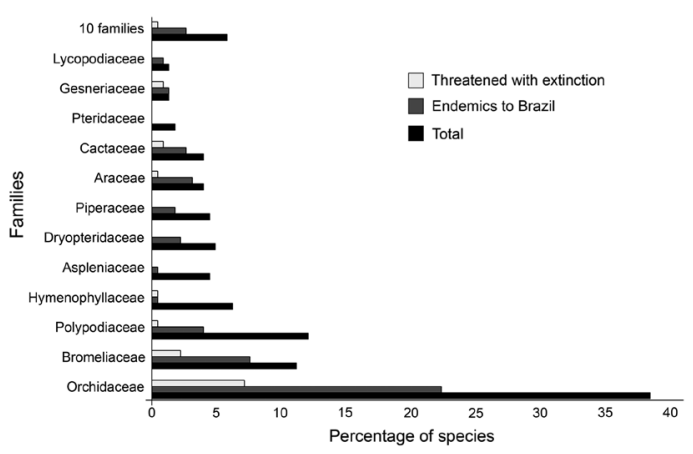

Figure 3 - Representativity of the families of vascular epiphytes and comparison with percentage of endemics and threatened with extinction in the cloud forest of Parque Estadual do Ibitipoca, Minas Gerais, Brazil.

ombrophilous forest in Brazil (Waechter 1992; Kersten \& Kunyioshi 2006; Blum et al. 2011; Lima et al. 2011). This richness is even more remarkable when accounting for the total area of this conservation unit $(1,488 \mathrm{ha})$, of which only $20 \%$ presents forest vegetation (i.e., less than 300 ha are composed of this physiognomy). The richness of vascular epiphytes in the PEIB also represents approximately $9.5 \%$ of the total found in the BAF $[2,256$ species according to Freitas et al. (2016)], in a conservation unit that comprises only $0.001 \%$ of this phytogeographic domain $(0.00085 \%$ if considering only the area covered by cloud forests).

Evolutionary lineages have a different configuration regarding the proportion identified in the BAF by Kersten (2010), with monocotyledons presenting a slightly smaller percentage $(54.3 \%$ versus $63.5 \%$ ) of that attributed to the BAF. A similar result was obtained for eudicotyledons (with $8.5 \%$ in PEIB and $14.1 \%$ in BAF). On the other hand, ferns have a higher diversity $(31.4 \%)$ in the PEIB than in the BAF $(16.4 \%)$.

The richest families found in the PEIB are among the richest often found in similar studies in several forest physiognomies in the Neotropical Region (Hietz \& Hietz-Seifert 1995; Dittrich et al. 1999; Kersten \& Silva 2001; Arévalo \& Betancur 2004; Giongo \& Waechter 2004; Kersten et al. 2009; Bianchi et al. 2012; Alves \& Menini Neto 2014; Barbosa et al. 2015), although the order can be different in some cases.

These families (Tab. 2) are also the main families of vascular epiphytes in the BAF, despite the differences in representativity. According to the data of Freitas et al. (2016), Araceae have a similar proportion in the BAF and PEIB, while Orchidaceae reaches $46.5 \%$ of all vascular epiphytes of the BAF and represents $38.6 \%$ of the total families in the PEIB. A similar result was found for Bromeliaceae, which represents $26.2 \%$ of the epiphytic species of the BAF and accounts for only $11.2 \%$ of the species in the PEIB. Thus, the reduced contribution of the last two families results in a smaller contribution of the monocotyledons to the epiphytic flora.

On the other hand, Polypodiaceae has greater representativity, with $12.1 \%$ of the vascular epiphytes of this conservation unit, a value substantially superior to the $4.3 \%$ found in the BAF (Freitas et al. 2016), contributing to the richness of ferns in the flora of the PEIB. The richness of this lineage was enhanced by the Hymenophyllaceae, (the fourth richest; $6.3 \%$ of total), which represents only $1.8 \%$ (in ninth position) in the BAF epiphytes, next to Aspleniaceae and Dryopteridaceae (5\% each) which both have a greater relative representativity in the PEIB than in the BAF (around 1.8\% each).

As reviewed by Kersten (2010), Orchidaceae is the richest family in the dense ombrophilous forest of the BAF, followed by Bromeliaceae, Araceae, Polypodiaceae, Cactaceae, Dryopteridaceae, Gesneriaceae, Hymenophyllaceae, and Piperaceae. It is apparent that although the richest families in the PEIB (Tab. 2, Fig. 2) are among the richest of this type of forest in the BAF, there is a reduced contribution of the angiosperm families Araceae, Cactaceae, and Gesneriaceae.

A comparison with some punctual studies of vascular epiphytes in ombrophilous and montane ombrophilous forests (Tab. 2) shows a consistent greater relative richness of ferns, with Polypodiaceae as the second richest in four of six sites (all areas with elevations superior to 1,000 $\mathrm{m})$. Hymenophyllaceae is well represented in all sites, and Aspleniaceae and Dryopteridaceae are also among the richest families in the majority of sites. On the other hand, there are fewer Araceae species in sites of high elevation, except in the Parque Estadual Carlos Botelho, which has a wide altitudinal gradient and lower altitudes of approximately $30 \mathrm{~m}$.

Thus, ferns with only marginal importance in the BAF or globally (e.g., Aspleniaceae, Dryopteridaceae and Hymenophyllaceae) contribute less than $2 \%$ of the total richness in both cases (Madison 1977; Kersten 2010; Zotz 2013; Freitas et al. 2016) in the PEIB have a greater 


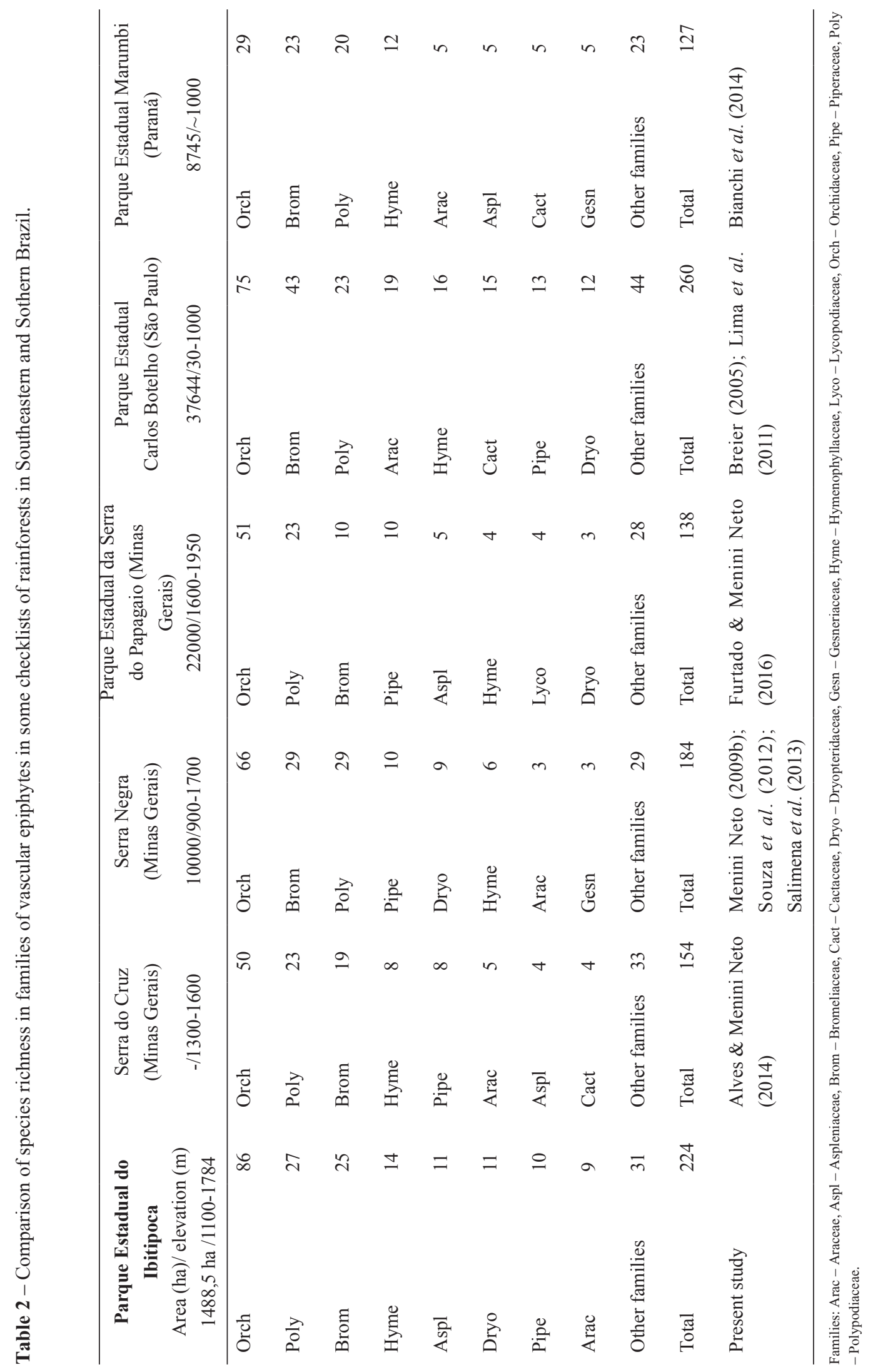


richness to the detriment of angiosperm families of vascular epiphytes often found among the richest. For example, Araceae, Piperaceae, and Cactaceae are the fourth, fifth and sixth richest families in the BAF, respectively; however, Cactaceae did not figure among the richest of the PEIB.

Elevation could contribute to this pattern, due to the sensitivity of some families to low temperatures. For example, Araceae has a lower percentage in the physiognomy of the mixed ombrophilous forests, which are common in the southern region of Brazil (Kersten 2010), occurring at higher latitudes (and, consequently, lower temperatures). Consistently, only one species of Araceae was observed by Furtado \& Menini Neto (2016) in a mixed ombrophilous forest in the Serra da Mantiqueira ranging from 1,600 to $1,650 \mathrm{~m}$ of elevation. Krömer et al. (2005) and Cardelús et al. (2006) also recorded a reduction in the richness of this family correlating with the enhancement of elevation in the Andes. On the other hand, ferns have a greater richness that correlates with enhanced elevation (Moran 1995; Hietz \& Hietz-Seifert 1995; Krömer et al. 2005; Cardelús et al. 2006). Further investigation in Brazilian mountainous areas will contribute to determining whether this is a consistent pattern associated with cloud forests.

The epiphytic quotient observed in the PEIB reinforces the relevance of epiphytes in the diversity of flora. This quotient is $15 \%$ in the BAF (Freitas et al. 2016), and this index varies from 13 to $32 \%$ (average of $\sim 20 \%$ ) in areas of Brazilian dense ombrophilous forests (Kersten 2010). Thus, the quotient percentage observed in the PEIB is higher than the average in the BAF and of the majority of areas where this value was calculated. The relevance of this value must be highlighted because it is the same as that of the Equator flora (Moller-Jorgensen \& León-Yánez 1999; Zotz 2016) and higher than the quotient for the flora of Panama (Foster \& Hubbel 1990), indicating the importance of this synusia to the cloud forest in the studied area.

The majority of the 75 recorded genera (excluding those completely accidental) are tropically distributed (34 Neotropical and 17 Pantropical), and only four genera are considered Cosmopolitan. Eight genera occur exclusively in the BAF, of which six are endemic to Brazil (Centroglossa Barb.Rodr., Dyssochroma Miers, Hatiora Britton \& Rose, Promenaea Lindl., Schlumbergera Lem., Thysanoglossa Porto \& Brade). Six genera are found in the BAF and
Cerrado (CE), of which three are endemic to Brazil (Grobya Lindl., Isabelia Barb.Rodr., Nematanthus Schrad.). In addition, Nidularium is endemic to Brazil, occurring in Caatinga (CA) and the BAF, and Wittrockia Lindm. is also endemic, but occurs in CA, CE, and BAF. Griselinia J.R. Forst. \& G. Forst present a disjunct distribution in South America and New Zealand, and Neoregelia L.B.Sm. is disjunct between the Andes/Amazonia (AM) and the BAF. Scuticaria Lindl. is found in AM, CE, BAF, and Racinaea M.A. Spencer \& L.B.Sm. in AM and AF.

Among the 111 endemic Brazilian species (BFG 2015; Prado et al. 2015), four are endemic to Minas Gerais: Anthurium leoni, Neoregelia oligantha, Hoffmannseggella crispata (accidental), and Peperomia decora. Among the angiosperms, 38 represent species not recorded in the PEIB by Menini Neto et al. (2009a), of which only six are accidental.

Thirteen species are threatened with extinction at the country level, of which five are classified as Near Threatened, four as Vulnerable, three as Endangered, and one as Critically Endangered (Martinelli \& Moraes 2013). There are 23 threatened species at the state level, of which seven are classified as Vulnerable, nine as Endangered, six as Critically Endangered, and one as Data Deficient (Drummond et al. 2008). Orchidaceae has the greatest number of threatened species (16 at the country or state level, or both), due in particular to the ornamental appeal of several species.

The conservation of epiphytic species represents a challenge due to habitat suppression, which threatens the biodiversity as a whole or due to the sensitivity of fragmentation and collection of ornamental species. Both the richness and the complexity of epiphyte communities in the PEIB, as well as the presence of species at different levels of risk and endemism, reinforces its importance for biodiversity, revealing that relatively small areas are also relevant to the preservation, and even consolidated conservation units must improve the strategies for maintaining the biodiversity.

\section{Acknowledgements}

We would like to thank the specialists who helped us with the identification of the species: ferns (Alexandre Salino, Thaís Elias Almeida, Claudine M. Mynssen, João Paulo Santos Condack, Lana da Silva Sylvestre, Vinicius Antônio Oliveira Dittrich), Araceae (Livia Godinho, Cassia Sakuragui, Marcus Nadruz Coelho), Bromeliaceae 
(Rafaela Campostrini Forzza, Raquel Monteiro), Cactaceae (Daniela Zappi, Diego Rafael Gonzaga), Gesneriaceae (Alain Chautems), Piperaceae (Erika Von Sohsten Medeiros, Elsie Franklin Guimarães, Daniele Monteiro); Instituto Estadual de Florestas of Minas Gerais (IEF-MG) and Programa de PósGraduação em Ecologia da Universidade Federal de Juiz de Fora (PGECOL/UFJF) for the logistic support; Coordenação de Aperfeiçoamento de Pessoal de Nível Superior (CAPES) for the study grant conceded to S.G. Furtado, and to Dr. Ana Paula Gelli de Faria and Dr. Rafaela Campostrini for the comments on the manuscript.

\section{References}

Alves FE \& Menini Neto L (2014) Vascular epiphytes in a forest fragment of Serra da Mantiqueira and floristic relationships with Atlantic high altitude areas in Minas Gerais. Brazilian Journal of Botany 37: 187-196.

Alves RJV, Kolbek J \& Becker J (2008) Vascular epiphyte vegetation in rocky savannas of southeastern Brazil. Nordic Journal of Botany 26: 101-117.

APG IV - The Angiosperm Phylogeny Group (2016) An update of the Angiosperm Phylogeny Group classification for the orders and families of flowering plants: APG IV. Botanical Journal of the Linnean Society 181: 1-20.

Arévalo R \& Betancur J (2004) Diversidad de epífitas vasculares en cuatro bosques del sector suroriental de la Serranía de Chiribiquete, Guayana Colombiana. Caldasia 26: 359-380.

Barbosa DEF, Basílio GA, Silva FR \& Menini Neto L (2015) Vascular epiphytes in a remnant of seasonal semideciduous forest in Zona da Mata of Minas Gerais Brazil. Bioscience Journal 31: 623-633.

Benzing DH (1990) Vascular epiphytes: general biology and related biota. Cambridge University Press, Cambridge. 376p.

BFG - The Brazil Flora Group (2015) Growing knowledge: an overview of seed plant diversity in Brazil. Rodriguésia 66: 1085-1113.

Bianchi JS, Michelon C \& Kersten RA (2012) Epífitas vasculares de uma área de ecótono entre as Florestas Ombrófilas Densa e Mista, no Parque Estadual do Marumbi, PR. Estudos de Biologia (Ambiente e Diversidade) 34: 37-44.

Blum CT, Roderjan CV \& Galvão F (2011) Composição florística e distribuição altitudinal de epífitas vasculares da floresta ombrófila densa na Serra da Prata, Morretes, Paraná, Brasil. Biota Neotropica 11: 141-159.

Breier TB (2005) O epifitismo vascular em florestas do sudeste do Brasil. Tese de Doutorado. Universidade Estadual de Campinas, São Paulo. 139p.
Cardelús CL, Colwell RK \& Watkins JE (2006) Vascular epiphyte distribution patterns: explaining the midelevation richness peak. Journal of Ecology 94: 144-156.

CETEC (1983) Diagnóstico ambiental do estado de Minas Gerais. Fundação Centro Tecnológico de Minas Gerais, Belo Horizonte. 158p.

Christenhusz MJM, Zhang X-C \& Schneider H (2011) A linear sequence of extant families and genera of lycophytes and ferns. Phytotaxa 19: 7-54

Costa CMR, Hermann G \& Martins CS (1998) Biodiversidade em Minas Gerais: um atlas para sua conservação. Fundação Biodiversitas, Belo Horizonte. 94p.

Dittrich VAO, Kozera C \& Silva SM(1999) Levantamento florístico dos epífitos vasculares do Parque Barigüi, Curitiba, Paraná, Brasil. Iheringia, série Botânica 52: 11-22.

Drummond GM, Martins CS, Machado ABM, Sebaio FA \& Antonini Y (orgs.) (2005) Biodiversidade em Minas Gerais, um atlas para sua conservação. $2^{\mathrm{a}}$ ed. Fundação Biodiversitas, Belo Horizonte. 222p.

Drummond GM, Machado ABM, Martins CS, Mendonça MP \& Stehmann JR (2008) Listas vermelhas das espécies da fauna e da flora ameaçadas de extinção em Minas Gerais. 2a ed. Fundação Biodiversitas, Belo Horizonte. CD-Rom. Available at $<$ http:// www.biodiversitas.org.br/cdlistavermelha/default. asp $>$ Access on 20 June 2015.

Forzza RC, Menini Neto L, Salimena FRG \& Zappi D (orgs.) (2013) Flora do Parque Estadual do Ibitipoca e seu entorno. Editora UFJF, Juiz de Fora. 382p.

Foster RB \& Hubbell SP (1990) The floristic composition of the Barro Colorado Island forest. In: Gentry AH (ed.). Four Neotropical rainforests. Yale University Press, New Haven. Pp. 85-98.

Freitas L, Salino A, Menini Neto L, Almeida TE, Mortara SR, Stehmann JR, Amorim AM, Guimarães EF, Coelho MN, Zanin A \& Forzza RC (2016) A comprehensive checklist of vascular epiphytes of the Atlantic Forest reveals outstanding endemic rates. PhytoKeys 58: 65-79.

Furtado SG \& Menini Neto L (2015a) Diversity of vascular epiphytes in two high altitude biotopes of the Brazilian Atlantic Forest. Brazilian Journal of Botany 38: 295-310.

Furtado SG \& Menini Neto L (2015b) Diversity of vascular epiphytes in urban environment: a case study in a biodiversity hotspot, the Brazilian Atlantic Forest. CES Revista 29: 82-101.

Furtado SG \& Menini Neto L (2016) Vascular epiphytic flora of a high montane environment of Brazilian Atlantic Forest: composition and floristic relationships with other ombrophilous forests. Acta Botanica Brasilica 30: 422-436. DOI: 10.1590/0102-33062016abb0090 
Gentry AH \& Dodson CH (1987) Diversity and biogeography of neotropical vascular epiphytes. Annals of the Missouri Botanical Garden 74: 205-223.

Giongo C \& Waechter JL (2004) Composição florística e estrutura comunitária de epífitos em uma floresta de galeria na Depressão Central do Rio Grande do Sul. Revista Brasileira de Botânica 27: 563-572.

Hietz P \& Hietz-Seifert U (1995) Composition and ecology of vascular epiphyte communities along an altitudinal gradient in Central Veracruz, Mexico. Journal of Vegetation Science 6: 487-498.

Hosokawa T (1950) Epiphyte-quotient. Botanical Magazine of Tokyo 63: 18-19.

Kersten RA (2010) Epífitas vasculares - histórico, participação taxonômica e aspectos relevantes com ênfase na Mata Atlântica. Hoehnea 37: 9-38.

Kersten RA \& Kunyioshi YS (2006) Epífitos vasculares na Bacia do Alto Iguaçu, Paraná, Brasil -composição florística. Estudos de Biologia 28: 55-71.

Kersten RA, Kuniyoshi YS \& Roderjan CV (2009) Epífitas vasculares em duas formações ribeirinhas adjacentes na bacia do rio Iguaçu - Terceiro Planalto Paranaense. Iheringia, Série Botânica 64: 33-43.

Kersten RA \& Silva SM (2001) Composição florística e distribuição espacial de epífitas vasculares em floresta da planície litorânea da Ilha do Mel, Paraná, Brasil. Revista Brasileira de Botânica 24: 213-226.

Körner C (2004) Mountain biodiversity, its causes and function. Ambio 13: 11-17. Available at $<$ http:// www.ncbi.nlm.nih.gov/pubmed/15575177>. Access on 15 September 2015.

Krömer T, Kessler M, Gradstein R \& Acebey A (2005) Diversity patterns of vascular epiphytes along an elevational gradient in the Andes. Journal of Biogeography 32: 1799-1809.

Lima RAF, Dittrich VAO, Souza VC, Salino A, Breier TB \& Aguiar OT (2011) Flora vascular do Parque Estadual Carlos Botelho, São Paulo, Brasil. Biota Neotropica 11: 173-214.

Luizi-Ponzo AP, Siviero TS, Amorim ET, Henriques DK, Rocha LM, Gomes HCS, Paiva LA, Rodrigues RS, Silva IC, Silva AGD, Ribeiro GC, Gomes CQ \& Campeão AS (2013) Briófitas do Parque Estadual do Ibitipoca no Herbário Prof. Leopoldo Krieger. In: Forzza RC, Menini Neto L, Salimena FRG \& Zappi D (orgs.) Flora do Parque Estadual do Ibitipoca e seu entorno. Editora UFJF, Juiz de Fora. Pp. 95-122.

Madison M (1977) Vascular epiphytes: their systematic occurrence and salient features. Selbyana 2: 1-13.

Martinelli G \& Moraes MA (orgs.) (2013) Livro vermelho da flora do Brasil. Jardim Botânico do Rio de Janeiro, Andrea Jakobsson, Rio de Janeiro. 1100 p.

Menini Neto L, Forzza RC \& Zappi D (2009a) Angiosperm epiphytes as conservation indicators in forest fragments: A case study from southeastern Minas Gerais, Brazil. Biodiversity and Conservation 18: 3785-3807.

Menini Neto L, Matozinhos CN, Abreu NL, Valente ASM, Antunes K, Souza FS, Viana PL \& Salimena FRG (2009b) Flora vascular não-arbórea de uma floresta de grota na Serra da Mantiqueira, Zona da Mata de Minas Gerais, Brasil. Biota Neotropica 9: 149-161.

Moller-Jorgensen P \& León-Yánez S (1999) Catalogue of the vascular plants of Ecuador. Missouri Botanical Garden Press, St. Louis. 1181p.

Moran RC (1995) The importance of mountains to pteridophytes, with emphasis on Neotropical montane forests. In: Churchill SP, Balslev H, Forero E \& Luteyn JL (eds.) Biodiversity and conservation of Neotropical montane forests (Proceedings of a symposium, New York Botanical Garden, 21-26 June 1993). New York Botanical Garden, New York. Pp. 359-363.

Oliveira-Filho AT, Fontes MAL, Viana PL, Valente ASM, Salimena FRG \& Ferreira FM (2013) O mosaico de fitofisionomias do Parque Estadual do Ibitipoca. In: Forzza RC, Menini Neto L, Salimena FRG \& Zappi D (orgs.) Flora do Parque Estadual do Ibitipoca e seu entorno. Editora UFJF, Juiz de Fora. Pp. 53-93.

PPG I (2016) A community-derived classification for extant lycophytes and ferns. Journal of Systematics and Evolution 54: 563-603.

Prado J, Sylvestre LS, Labiak PH, Windisch PG, Salino A, Barros ICL, Hirai RY, Almeida TE, Santiago ACP, Kieling-Rubio MA, Pereira AFN, Øllgaard B, Ramos CGV, Mickel JT, Dittrich VAO, Mynssen CM, Schwartsburd PB, Condack JPS, Pereira JBS \& Matos FB (2015) Diversity of ferns and lycophytes in Brazil. Rodriguésia 66: 1073-1083.

Salimena FRG, Matozinhos CN, Abreu NL, Ribeiro JH, Souza FS \& Menini Neto L (2013) Flora fanerogâmica da Serra Negra, Minas Gerais, Brasil. Rodriguésia 64: 311-320.

Salino A, Almeida TE, Mynssen CM, Condack JPS \& Sylvestre LS (2013) Pteridófitas do Parque Estadual do Ibitipoca. In: Forzza RC, Menini Neto L, Salimena FRG \& Zappi D (orgs.) Flora do Parque Estadual do Ibitipoca e seu entorno. Editora UFJF, Juiz de Fora. Pp. 123-152.

Souza FS, Salino A, Viana PL \& Salimena FRG (2012) Pteridófitas da Serra Negra, Minas Gerais, Brasil. Acta Botanica Brasilica 26: 378-390.

Thiers B [continuously updated]. Index herbariorum: a global directory of public herbaria and associated staff. The New York Botanical Garden. Available at $<$ http://sweetgum.nybg.org/science/ih/>. Access on 20 June 2016.

Waechter JL (1992) O epifitismo vascular na planície costeira do Rio Grande do Sul. Tese de Doutorado. 
Universidade Federal de São Carlos, São Carlos. $163 p$.

Werneck M \& Espírito-Santo MM (2002) Species diversity and abundance of vascular epiphytes on Vellozia piresiana in Brazil. Biotropica 34: 51-57.
Zotz G (2013) The systematic distribution of vascular epiphytes - a critical update. Botanical Journal of the Linnean Society 171: 453-481.

Zotz G (2016) Plants on plants - the biology of vascular epiphytes. Springer, Cham. 282p. 
